\title{
Higher-Order Approximate Solutions of Neumann Problems by Isoparametric Finite Element Method with Relevant Lumping Operator
}

\author{
$\mathrm{BY}$ \\ Masahisa TABATA*
}

\section{§ 1. Introduction}

In 1966, Friedrichs and Keller [1] proposed a generalized finite difference scheme for the Neumann problem. Today, their method can be regarded as a finite element method using the piecewise linear "chajeau" bases. From the riew point of computations, however, their method is not convenient enough, except the case of a polygonal domain, since it requires to perform all the integrations which appear in obtaining Ritz coefficients and right-hand sides of determinate linear equations, in an exact domain and on an exact boundary.

The purpose of this paper is to consider a higher-order approximate procedure for the Neumann problem in an approximate domain, using isoparametric finite elements. This forces us to change slightly the data functions to guarantee the solvability of the determinate linear system. We shall study some kinds of errors, which are caused by the selection of the basis functions (approximation crrors), by the change in domain, by the approximation of data functions and b! the change of data functions for the solvability. Frrors caused by the selection of the basis functions are, of course, inevitable. It is desirable that crrors due to other reasons mentioned above do not destroy the order of accuracy which the basis functions could achieve. In this sense, it appears to be reasonable to expect that, when the boundary $\Gamma$ is curved, the optimal order of accuracy can be achieted by the use of isoparametric technique. Also, we may apply the lumping technique to the body force term $f$, which may simplify

Communicated by H. Yoshizawa, August 17, 1974. Revised April 1, 1976.

* Department of Mathematics, Faculty of Science, Kyoto University, Kyoro 606, Japan. 
the computation of the inner product including it. Our main conclusion is that the scheme using the isoparametric elements guarantees the optimal order of accuracy in $H^{1}$-norm, and that it may not be destroyed by the introduction of a class of lumping operators. $L^{2}$-estimates are also obtained using Nitsche's trick [2]. Our results can be extended also to the Dirichlet problem straightforwards.

Strang and Fix [8] have discussed the error due to the change in domain for the equation $-\Delta u+u=f$ with the Neumann condition, in which there is no problem on the solvability of approximate equations. With regards to the Dirichlet problem, Ciarlet and Raviart [4], Nitsche [3], and Strang and Verger [7] have investigated the effects of approximate boundary conditions. Numerical integrations, which may produce errors of different type, are also taken into account in [4]. Aubin [10] has discussed external approximations from the theoretical standpoint. The lumping operator in this paper may also be considered as one of them.

In $\S 2$, we introduce the problem. In $\S 3$, we obtain general error estimates for approximate solutions with a lumping operator in an approximate domain. In $\S 4$, after the isoparametric finite element procedure and the relevant lumping operator are described in detail, the convergence orders are obtained in terms of the maximum side-length of the triangles. Although the isoparametric finite elements we treat here are of Lagrangean type only, it may be clear that we can extend the results to the case of elements of Hermitian type. In the last section, we show some examples. For the results of the numerical experiments of these examples, readers are referred to [12].

\section{§ 2。 Prelliminaries}

The problem we consider is

$$
\begin{cases}-\Delta u=f & \text { in } \Omega, \\ \frac{d u}{d n}=g & \text { on } \Gamma,\end{cases}
$$

where $\Omega$ is a bounded domain in $\mathbb{R}^{2}$ and $\Gamma$ is its boundary. We assume that $\Gamma$ is sufficiently smooth and that it is expressed as

$$
\Gamma=\left\{(X(t), Y(t)) ; \dot{X}(t)^{2}+\dot{Y}(t)^{2} \equiv 1\right\} .
$$


In $(2 \cdot 1), \Delta=\partial^{2} / \partial x^{2}+\partial^{2} / \partial y^{2}$ and $n$ refers to the outer normal to $\Gamma$. Let $H^{m}(\Omega)$ denote the usual Sobolev space with the norm given by

$$
\|u\|_{m, \Omega}=\left\{\sum_{|\alpha| \leq m} \int_{\Omega}\left|D^{\alpha} u\right|^{2} d x d y\right\}^{1 / 2}
$$

Here $\alpha=\left(\alpha_{1}, \alpha_{2}\right)$, and $\alpha_{1}$ and $\alpha_{2}$ are non-negative integers such that

$$
|\alpha|=\alpha_{1}+\alpha_{2} \quad \text { and } \quad D^{\alpha} u=\frac{\partial^{|\alpha|} u}{\partial x^{\alpha_{1}} \partial y^{\alpha_{2}}}
$$

$H^{0}(\Omega)$ is also denoted by $L^{2}(\Omega)$. Similarly, $H^{m}(\Gamma)$ and $L^{2}(\Gamma)$ are defined to be the Sobolev spaces defined on $\Gamma$. Here and later, $c$ indicates a generic numerical positive constant, independent of $h, u, f$ and $g$, which may be different at different places. ( $h$ is a parameter depending on a subdivision of $\Omega$.)

An approximate domain $\Omega_{h}$ is obtained as a union of finite elements. ( $\Omega_{h}$ is not always a polygonal domain. For details, see $\S 4$.) Let $\left\{\mathrm{P}_{i}\right\}_{i=1}^{N_{1}}$ be all the nodal points in $\bar{\Omega}_{h}$. Let $S\left(\Omega_{h}\right)$ be a subspace of $H^{1}\left(\Omega_{h}\right)$ spanned by $\left\{\widehat{\phi}_{i}(x, y)\right\}_{i=1}^{N_{1}}$ such that

$$
\begin{gathered}
\widehat{\phi}_{i} \in C\left(\bar{\Omega}_{h}\right) \cap H^{1}\left(\Omega_{h}\right), \\
\widehat{\phi}_{i}\left(\mathrm{P}_{j}\right)=\delta_{i j} \quad \text { for } \quad i, j=1, \cdots, N_{1},
\end{gathered}
$$

and

$$
\sum_{i=1}^{N_{1}} \widehat{\phi}_{i} \equiv 1 \quad \text { in } \Omega_{h} \text {. }
$$

Let $T\left(\Omega_{h}\right)$ be a subspace of $L^{2}\left(\Omega_{h}\right)$ spanned by $\left\{\bar{\phi}_{i}(x, y)\right\}_{i=1}^{N_{2}}\left(N_{2} \leqq N_{1}\right)$ such that

$$
\bar{\phi}_{i} \in L^{2}\left(\Omega_{n}\right)
$$

and

$$
\sum_{i=1}^{N_{2}} \bar{\phi}_{i} \equiv 1 \quad \text { almost everywhere in } \Omega_{h} \text {. }
$$

We define two linear mappings $P$ and $Q$ from $C\left(\bar{\Omega}_{h}\right)$ into $S\left(\Omega_{h}\right)$ and $T\left(\Omega_{h}\right)$, respectively,

$$
\begin{aligned}
& P: C\left(\bar{\Omega}_{h}\right) \rightarrow S\left(\Omega_{h}\right) \quad \text { such that } \\
& P u=\sum_{i=1}^{N_{1}} u\left(\mathrm{P}_{i}\right) \widehat{\phi}_{i}, \\
& Q: C\left(\bar{\Omega}_{h}\right) \rightarrow T\left(\Omega_{h}\right) \quad \text { such that }
\end{aligned}
$$




$$
Q u=\sum_{i=1}^{N_{2}} u\left(Q_{i}\right) \bar{\phi}_{i},
$$

where $\left\{Q_{i}\right\}_{i=1}^{N_{2}}$ is a subset of $\left\{\mathrm{P}_{i}\right\}_{i=1}^{N_{1}}$. $Q$ is called a lumping operator associated with $P$.

Remark 2.1. For $\bar{\phi}_{i}$, the condition as $(2.4)$ is not required.

Remark 2.2. If we take $\bar{\phi}_{i}=\widehat{\phi}_{i}$ and $Q_{i}=\mathrm{P}_{i}\left(i=1, \cdots, N_{2}=N_{1}\right)$, then $Q=P$.

Remark 2.3. $P^{2}=P$ and $Q P=Q$.

Remark 2.4. We assume that $\Gamma_{h}$ (the boundary of $\Omega_{h}$ ) approximates $\Gamma$ so naturally that the following conditions are satisfied:

i) There exists a bounded domain $\widetilde{\Omega}$ such that $\bar{\Omega} \subset \widetilde{\Omega}$ and $\bar{\Omega}_{h} \subset \widetilde{\Omega}$ for al] $h$,

ii) for any $f \in H^{1}\left(\Omega_{h}\right)$, there exists a $\tilde{f} \in H^{1}(\widetilde{\Omega})$ such that

$$
\|\tilde{f}\|_{i, \tilde{\Omega}} \leqq c\|f\|_{i, \Omega_{h}} \quad \text { for } \quad i=0,1,
$$

and

iii) for any $f \in H^{1}\left(\Omega_{h}\right)$, it holds that

$$
\|f\|_{0, \Gamma_{h}} \leqq c\|f\|_{1, \Omega_{h}} .
$$

These conditions may be satisfied when $\Omega$ is approximated by a polygon $\Omega_{h}$ whose vertices are all on $\Gamma$, or when isoparametric finite element procedures are used.

We use the following notations throughout this paper:

$$
\begin{gathered}
(f, v)=\int_{\Omega} f \cdot v d x d y \quad \text { for } f, v \in L^{2}(\Omega), \\
a(u, v)=(\partial u / \partial x, \partial v / \partial x)+(\partial u / \partial y, \partial v / \partial y) \text { for } u, v \in H^{1}(\Omega), \\
{[g, v]=\int_{\Gamma} g \cdot v d t \text { for } g, v \in L^{2}(\Gamma)}
\end{gathered}
$$

and

$$
|\nabla u|=\left\{|\partial u / \partial x|^{2}+|\partial u / \partial y|^{2}\right\}^{1 / 2} \text { for } u \in H^{1},
$$

and we define $(f, v)_{h}, a_{h}(u, v)$ and $[g, v]_{h}$ by replacing $\Omega$ and $\Gamma$ with $\Omega_{h}$ and $\Gamma_{h}$ in $(f, v), a(u, v)$ and $[g, v]$, respectively.

\section{§ 3. Gemeral Erom Estimates for Approximate Solutions}

In order that a solution of $(2 \cdot 1)$ exists, the condition on $f$ and $g$, 


$$
(f, 1)+[g, l]=0,
$$

is required. Conversely, if $f$ and $g$ satisfy (3-1) and have appropriatc smoothness, a solution exists in $H^{m}(\Omega)$ uniquely under the condition

$$
(u, 1)=0 \text {, }
$$

for some $m$. Let $\bar{\imath} \in H^{m}(\widetilde{\Omega})$ be a smooth extension of $u$ such that

$$
\|\widetilde{u}\|_{m, \hat{\Omega}} \leqq c\|u\|_{m, \Omega},
$$

and $u_{1}$ be $\widetilde{u}-k_{1}$, where

$$
k_{1}=(\widetilde{u}, 1)_{h} / \operatorname{mes}\left(\Omega_{h}\right) .
$$

We give two functions $f_{A} \in L^{2}\left(\Omega_{h}\right)$ and $g_{A} \in L^{2}\left(\Gamma_{h}\right)$, which may approximate $f$ and $g$ in some sense. (An example of $f_{A}$ and $g_{A}$ will be given later in Theorem 4.1.) Let $f_{1}$ be $f_{A}-k_{2}$, where

$$
k_{2}=\left\{\left(f_{A}, 1\right)_{h}+\left[g_{1}, 1\right]_{h}\right\} / \operatorname{mes}\left(\Omega_{h}\right) .
$$

The finite element solution of $(2 \cdot 1)$ and $(3 \cdot 2)$ is defined to be the function $\hat{u} \in S\left(\Omega_{h}\right)$ such that

$$
a_{h}(\hat{u}, \hat{\phi})=\left(f_{1}, Q \hat{\phi}\right)_{h}+\left[g_{A}, \phi\right]_{h} \quad \text { for } \quad \forall \hat{\phi} \in S\left(\Omega_{n}\right)
$$

and

$$
(i, 1)_{h}=0 \text {. }
$$

Theorem 3. 1. We assume that $\Gamma_{b}$ is a natural approximation of $\Gamma$ in the sense of Remark 2.4. Further, we assume (2.3), (2.4) and (2.5) for $S\left(\Omega_{h}\right)$, and $(2 \cdot 6)$ and $(2 \cdot 7)$ for $T\left(\Omega_{h}\right)$. Then, the syslom (3.6) and (3.7) has a unique solution $u$ in $S\left(\Omega_{h}\right)$, and

$$
\begin{aligned}
& \|\tilde{u}-\widetilde{u}\|_{1, \Omega_{h}} \leqq c\left\{\left|k_{1}\right|+\|P \tilde{u}-\tilde{u}\|_{1, a_{h}}+\left(\left|k_{2}\right|+\left\|f_{1}+\Delta \widetilde{u}\right\|_{0, \Omega_{h}}\right)\right. \\
& \quad \times \frac{\|Q(\hat{u}-P \widetilde{u})\|_{0, \Omega_{h}}}{\|\hat{u}-P \widetilde{u}\|_{1, \Omega_{h}}}+\frac{\left|(\Delta \widetilde{u},(I-Q)(\dot{u}-P \widetilde{u}))_{h}\right|}{\|\hat{u}-P \widetilde{u}\|_{1, \Omega_{h}}} \\
& \left.+\left\|g_{A}-\frac{d \tilde{u}}{d n_{h}}\right\|_{0, I_{h}^{\prime}}\right\},
\end{aligned}
$$

where $n_{h}$ refers to the outer normal to $\Gamma_{h}$.

For the proof of Theorem 3.1, the following lemma is used. 
Lemma 3. 1. Let $E(\Omega)$ be a subspace of $H^{1}(\Omega)$ such that

$$
E(\Omega)=\left\{u \in H^{1}(\Omega) ;(u, 1)=0\right\} .
$$

Then, $\{a(u, u)\}^{1 / 2}$ is equivalent to $\|u\|_{1, \Omega}$ in $E(\Omega)$.

Proof. It is sufficient to show

$$
\|u\|_{0, \Omega} \leqq c|u|_{A} \quad \text { for } \quad \forall u \in E(\Omega),
$$

where $|u|_{A}=\{a(u, u)\}^{1 / 2}$. Let $k$ be any constant. It holds that

$$
(u, u)=(u, u-k) \leqq\|u\|_{o, \Omega}\|u-k\|_{0, \Omega} \quad \text { for } \quad \forall u \in E(\Omega) .
$$

Then we have

$$
\|u\|_{0, \Omega} \leqq \inf _{k}\|u-k\|_{0, \Omega} \leqq c|u|_{A}
$$

Here, the last inequality follows from the fact that $|u|_{A}$ is equivalent to the usual norm of the quotient space $H^{1}(\Omega) / P_{0}$, where $P_{0}$ is the set of all the constant functions. (See [11].)

Proof of Theorem 3.1. (3.6) forms $N_{1}$ linear equations,

$$
a_{h}\left(\hat{u}, \hat{\phi}_{i}\right)=\left(f_{1}, Q \widehat{\phi}_{i}\right)_{h}+\left[g_{A}, \widehat{\phi}_{i}\right]_{h} \quad \text { for } \quad i=1, \cdots, N_{1} .
$$

Summing up these $N_{1}$ equations, we obtain a trivial relation

$$
a_{h}(\widehat{u}, 1)=\left(f_{1}, 1\right)_{h}+\left[g_{A}, 1\right]_{h}=0
$$

from $(2 \cdot 5),(2 \cdot 7)$ and $(3 \cdot 5)$. Therefore, the system $(3 \cdot 6)$ and $(3 \cdot 7)$ is not overdetermined. Suppose that there exists a nontrivial solution $\widehat{u}$ for $f_{A}=g_{A}=0$. Then,

$$
a_{h}(\hat{u}, \hat{u})=0 \quad \text { and } \quad(\hat{u}, 1)_{h}=0 .
$$

These conclude $u \equiv 0$ by Lemma 3.1 , which is a contradiction. This shows that the system $(3 \cdot 6)$ and $(3 \cdot 7)$ is solvable for any data $f_{A}$ and $g_{A}$ and that it has a unique solution in $S\left(\Omega_{h}\right)$.

Let us prove $(3 \cdot 8)$. First,

$$
\|\widehat{u}-\widetilde{u}\|_{1, \Omega_{h}} \leqq\left\|\hat{u}-u_{1}\right\|_{1, \Omega_{h}}+c\left|k_{1}\right| .
$$

By Lemma 3.1, we have

$$
\left\|\hat{u}-u_{1}\right\|_{1, s_{h}}^{2} / c \leqq a_{h}\left(\hat{u}-u_{1}, \widehat{u}-u_{1}\right)
$$




$$
\begin{aligned}
& =a_{h}(\hat{u}-\widehat{u}, \hat{u}-\hat{u}) \\
& =a_{h}(u-\grave{u}, P \hat{u}-\bar{u})+a_{h}(\grave{u}-\widehat{u}, \grave{u}-P \hat{u}) .
\end{aligned}
$$

Each term of the right-hand side is estimated as follows:

$$
\begin{aligned}
& \mid 1 \text { st } \operatorname{term} \mid \leqq\|\widehat{u}-\widetilde{u}\|_{1, \Omega_{h}}\|P \widetilde{u}-\widetilde{u}\|_{1, \Omega_{h}}, \\
& (3 \cdot 16) \quad \text { 2nd term }|=|\left(f_{1}, Q(\hat{u}-P \widehat{u})\right)_{h}+\left[g_{A}, \widehat{u}-P \widehat{u}\right]_{h} \\
& -(-\Delta \widetilde{u}, \hat{u}-P \widetilde{u})_{h}-\left[\frac{d \widetilde{u}}{d n_{h}}, \hat{u}-P \widetilde{u}\right]_{h} \\
& \leqq\left|\left(f_{1}+\Delta \widetilde{u}, Q(\hat{u}-P \widetilde{u})\right)_{h}\right|+\left|(\Delta \widetilde{u},(I-Q)(\tilde{u}-P \widetilde{u}))_{h}\right| \\
& +\left|\left[g_{A}-\frac{d \widetilde{u}}{d n_{h}}, u-P \widetilde{u}\right]_{h}\right| \\
& \leqq\left\|f_{1}+\Delta \widetilde{u}\right\|_{0, \Omega_{h}}\|Q(\widehat{u}-P \widetilde{u})\|_{0, \Omega_{h}}+\left|(\Delta \widetilde{u},(I-Q)(\widehat{u}-P \widetilde{u}))_{h}\right| \\
& +c\left\|g_{A}-\frac{d \widetilde{u}}{d n_{h}}\right\|_{0, \Gamma_{h}}\|\widehat{u}-P \widetilde{u}\|_{1, s_{h}} .
\end{aligned}
$$

Combining $(3 \cdot 13) \sim(3 \cdot 16)$ and the trivial inequality

$$
\|\tilde{u}-P \widetilde{u}\|_{1, \Omega_{h}} \leqq\|u-\widehat{u}\|_{1, \Omega_{h}}+\|\breve{u}-P \widetilde{u}\|_{1, \Omega_{h}},
$$

we obtain $(3 \cdot 8)$. This completes the proof.

Let us estimate $\|\widehat{u}-\widetilde{u}\|_{0, s_{h}}$, using Nitsche's trick. Let $e$ be $\dot{u}-\widehat{u}$ $\in H^{1}\left(\Omega_{h}\right), \tilde{e} \in H^{1}(\widetilde{\Omega})$ be a smooth extension of $e$ such that

$$
\|\bar{c}\|_{i, \tilde{a}} \leqq c\|e\|_{i, \Omega_{h}} \quad \text { for } \quad i=0,1
$$

and $e_{1}$ be $\tilde{e}-k_{3}$, where

$$
k_{3}=(1, \dot{e}) / \operatorname{mes}(\Omega)
$$

Let $w \in H^{s}(\Omega)$ be the unique solution of

$$
\begin{cases}-J w=c_{1} & \text { in } \Omega, \\ \frac{d w}{d n}=0 & \text { on } \quad \Gamma, \\ (w, 1)=0, & \end{cases}
$$

and $\widetilde{w} \in H^{2}(\widetilde{\Omega})$ be a smooth extension of $w$ such that

$$
\|\widetilde{w}\|_{2, \tilde{\Omega}} \leqq c\|w\|_{2, \Omega}
$$


Theorem ${ }_{2}^{2}$ 2. In the same assumptions of Theorem 3.1. the following estimate is oblained,

$$
\begin{aligned}
& \|\widehat{u}-\widetilde{u}\|_{0, \Omega_{h}} \leqq c\left\{\|e\|_{1, \Omega_{n}} \frac{\|\widetilde{w}-P \widetilde{w}\|_{1, \Omega_{h}}}{\|\widetilde{w}\|_{2, \Omega_{h}}}+\left(\left\|f_{A}+\Delta \widetilde{u}\right\|_{0, \Omega_{h}}+\left|k_{2}\right|\right)\right. \\
& \quad \times \frac{\|Q \widetilde{w}\|_{0, \Omega_{h}}}{\|\widetilde{w}\|_{2, \Omega_{h}}}+\frac{\left|(\Delta \widetilde{u},(P-Q) \widetilde{w})_{h}\right|}{\|\widetilde{w}\|_{2, \Omega_{h}}}+\left\|g_{A}-\frac{d \widetilde{u}}{d n_{h}}\right\|_{0, \Gamma_{h}} \\
& \left.\quad \times \frac{\|P \widetilde{w}-\widetilde{w}\|_{1, \Omega_{h}}}{\|\widetilde{w}\|_{2, \Omega_{h}}}+\frac{\left|\left[g_{A}-\frac{d \widetilde{u}}{d n_{h}}, \widetilde{w}\right]_{h}\right|}{\|\widetilde{\mathfrak{w}}\|_{2, \Omega_{h}}}+\frac{J_{1}}{\|\widetilde{w}\|_{2, \Omega_{h}}}+J_{2}+\left|k_{3}\right|\right\},
\end{aligned}
$$

where $J_{1}=\int_{\Omega-\Omega_{h}}|\nabla \tilde{e}||\nabla \mathfrak{w}| d x d y+\int_{\Omega_{h}-\Omega}|\nabla c||\nabla \widetilde{\mathcal{W}}| d x d y$ and $J_{2}=\|e\|_{0, \Omega_{h}-\Omega}$ $+\|\tilde{e}\|_{0, s-\Omega_{h}}$.

Proof. From $(3 \cdot 20)$ and $(3 \cdot 21)$, we obtain

$$
\|\widetilde{w}\|_{2, \Omega_{h}} \leqq c\left\|e_{1}\right\|_{0, \Omega}
$$

and

$$
\begin{aligned}
\left\|e_{1}\right\|_{0, \Omega}^{2}= & \left(e_{1},-j w\right) \\
= & a\left(e_{1}, w\right) \\
= & a_{h}\left(e_{1}, \widetilde{w}-P \widetilde{w}\right)+a_{h}\left(e_{1}, P \widetilde{w}\right)+(\nabla \widetilde{e}, \nabla w)_{\Omega-\Omega_{h}} \\
& -(\nabla e, \nabla \widetilde{w})_{a_{h}-\Omega} .
\end{aligned}
$$

Each term of the right-hand side is estimated as follows:

$(3 \cdot 25) \quad \mid 1$ st term $\mid \leqq\|e\|_{1, \Omega_{h}}\|\widetilde{\boldsymbol{w}}-P \widetilde{\boldsymbol{v}}\|_{1, \Omega_{h}}$,

$(3 \cdot 26) \quad$ 2nd term $|=|\left(f_{1}, Q P \widetilde{\mathfrak{w}}\right)_{h}+\left[g_{A}, P \widetilde{\mathfrak{U}}\right]_{h}$

$$
\begin{aligned}
& \quad-(-\Delta \widetilde{u}, P \widetilde{w})_{h}-\left[\frac{d \widetilde{u}}{d n_{h}}, P \widetilde{w}\right]_{h} \mid \\
& =\mid\left(f_{1}+\Delta \widetilde{u}, Q \widetilde{w}\right)_{h}+(\Delta \widetilde{u},(P-Q) \widetilde{w})_{h} \\
& \quad+\left[g_{A}-\frac{d \widetilde{u}}{d n_{h}}, P \widetilde{w}-\widetilde{w}\right]_{h}+\left[g_{A}-\frac{d \widetilde{u}}{d n_{h}}, \widetilde{w}\right]_{h} \mid \\
& \leq c\left\|f_{1}+\Delta \widetilde{u}\right\|_{0, a_{h}}\|Q \widetilde{w}\|_{0, \Omega_{h}}+\left|(\Delta \widetilde{u},(P-Q) \widetilde{w})_{h}\right| \\
& \quad+c\left|g_{A}-\frac{d \widetilde{u}}{d n_{h}}\left\|_{0, \Gamma_{h}}\right\| P \widetilde{w}-\widetilde{w} \|_{1, \Omega_{h}}+\right|\left[g_{A}-\frac{d \widetilde{u}}{d n_{h}}, \widetilde{w}\right]_{h} \mid,
\end{aligned}
$$


and

$$
\mid 3 \text { rd term }+4 \text { th term } \mid \leqq J_{1} .
$$

Combining $(3 \cdot 23) \sim(3 \cdot 27)$ and the inequality

$$
\begin{aligned}
\|\boldsymbol{e}\|_{0, \Omega_{h}} & \leqq\|\tilde{e}\|_{0, \Omega}+c J_{2} \\
& \leqq\left\|e_{1}\right\|_{0 . \Omega}+c\left\{J_{2}+\left|k_{3}\right|\right\},
\end{aligned}
$$

we obtain $(3 \cdot 22)$. This completes the proof.

\section{\$4. Isoparamelric Finite Elements and Relevant Lumping Operators}

We shall illustrate the isoparametric finite element procedure. Let $T_{0}$ he a closed fundamental triangle with vertices $A_{1}(0,1), A_{2}(0,0)$ and $A_{3}(1,0)$ in $(\xi, \eta)$-plane. In $T_{0}$, there exist $k$ fundamental nodal points, $\mathrm{A}_{i}, i$ $=1, \cdots, k$, including the three vertices. Let $\left\{\widehat{\psi}_{i}(\xi, \eta)\right\}_{i-1}^{k}$ be a set of real functions defined in $T_{0}$ such that

i) $\hat{\psi}_{i} \in C\left(T_{0}\right) \cap H^{1}\left(T_{0}^{\circ}\right)$, where $T_{0}{ }^{\circ}$ is an interior of $T_{0}$,

ii) $\bar{\psi}_{i}\left(\mathrm{~A}_{j}\right)=\hat{o}_{i j}$ for $i, j=1, \cdots, k$,

iii) $\sum_{i=1}^{k} \widehat{\psi}_{i} \equiv 1$,

iv) there exist two scts of real numbers $\left\{\alpha_{i}\right\}_{i=1}^{k}$ and $\left\{\beta_{i}\right\}_{i=1}^{k}$ such that

$$
\sum_{i=1}^{k} \alpha_{i} \bar{\psi}_{i} \equiv \xi \text { and } \sum_{i=1}^{k} \beta_{i} \widehat{\psi}_{i} \equiv \eta,
$$

v) $\bar{\psi}_{i}=0$ on any side which does not contain $\mathrm{A}_{i}$, and

vi) the set $\left\{\left(\mathrm{A}_{i}, \widehat{\psi}_{i}\right) ; \mathrm{A}_{i}\right.$ is on the side of $\left.T_{0}\right\}$ is symmetric with respect to the barycenter of $T_{0}$, i.e., if $\mathrm{A}_{i}=\left(\lambda_{1}{ }^{i}, \lambda_{2}{ }^{i}, \lambda_{3}{ }^{i}\right)^{T}, \hat{\psi}_{i}(\lambda)$ $=\widehat{\psi}_{i}\left(\left(\lambda_{1}, \lambda_{2}, \lambda_{3}\right)^{T}\right)$ by the barycentric coordinate expression and $\lambda_{1}{ }^{i} \lambda_{2}{ }^{i}$ $\cdot \lambda_{3}{ }^{i}=0$, then $\left(S \lambda^{i}, \widehat{\psi}_{i}\left(S^{-j} \lambda\right)\right)$ coincides with some nodal point and its corresponding function, where $S$ is any $3 \times 3$ permutation matrix.

We triangulate $\Omega$ to obtain the set of closed triangles $\left\{d_{i}\right\}_{i=1}^{N_{T}}$ and the set of vertices $\left\{\mathrm{P}_{i}\right\}_{i-1}^{N_{0}}$ such that

i) $\mathrm{P}_{i} \in \bar{\Omega}$,

ii) $\Delta_{i} \cap \Delta_{j}$ is empty or equal to a common completely overlapping side, for any $i \neq j$.

iii) $\left.\inf _{i}\{\text { minimum angle of }\rfloor_{i}\right\} \geqq c$.

and 
iv) all rertices of the polygon $\bigcup_{i=1}^{N_{T}} \Delta_{i}$ are on $\Gamma$.

With each triangulation, $h$ is associated as follows:

$$
h=\max _{i}\left\{\text { maximum side-length of } \Delta_{i}\right\} .
$$

$\left\{A_{i}\right\}_{i=1}^{N_{T}}$ consists of two types of triangles, i.e., interior triangles and boundary triangles. An interior triangle has at most one vertex on $\Gamma$ and a boundary triangle has just two vertices on $\Gamma$. We add required nodal points $\left\{\mathrm{P}_{i}\right\}_{i=N_{0}+1}^{N_{1}}$ in $\bar{\Omega}$ so that there exist $k$ nodal points $\left\{\mathrm{B}_{i}^{j}\right\}_{i=1}^{k}$ in a neighbourhood of each triangle $d_{j}$ satisfying the following four conditions. i) $\quad\left\{\mathrm{B}_{i}^{j}\right\}_{i=1}^{k}$ is a subset of $\left\{\mathrm{P}_{i}\right\}_{i=1}^{N_{1}}$.

ii) $\mathrm{B}_{1}{ }^{j}, \mathrm{~B}_{2}{ }^{j}$ and $\mathrm{B}_{3}{ }^{j}$ are vertices of $d_{j}$.

iii) For the interior triangle, $\mathrm{B}_{i}{ }^{j}=\widetilde{\mathrm{B}}_{i}{ }^{j}$ for $i=4, \cdots, k$, where $\widetilde{\mathrm{B}}_{i}{ }^{j}$ is a point which has the same barycentric coordinates as $\mathrm{A}_{i}$.

iv) For the boundary triangle, $\mathrm{B}_{1}^{j}$ and $\mathrm{B}_{3}^{j}$ are on $\Gamma$. $\left(\mathrm{B}_{2}^{j}\right.$ is in $\Omega$.) $\mathrm{B}_{i}{ }^{j}=\widetilde{\mathrm{B}}_{i}{ }^{j}$ for all $\mathrm{A}_{i}$ lying on $\mathrm{A}_{1} \mathrm{~A}_{2}$ and $\mathrm{A}_{2} \mathrm{~A}_{3}$. For each $\mathrm{A}_{i}$ on $\mathrm{A}_{3} \mathrm{~A}_{1}, \mathrm{~B}_{i}{ }^{j}$ is taken on $\Gamma$ near the point $\mathrm{C}_{i}{ }^{j}$, which is on $\Gamma$ and satisfies

$$
\widehat{\mathrm{B}_{3}{ }^{j} \mathrm{C}_{i}^{j}}: \widehat{\mathrm{C}_{i}^{j} \mathrm{~B}_{1}^{j}}=\overline{\mathrm{A}_{3} \mathrm{~A}_{i}}: \overline{\mathrm{A}_{i} \mathrm{~A}_{1}},
$$

where $\widehat{\mathrm{B}}_{l}{ }^{j} \mathrm{C}_{i}^{j}$ is the length from $\mathrm{B}_{l}^{j}$ to $\mathrm{C}_{i}^{j}$ along $\Gamma$. Each $\mathrm{B}_{i}^{j}$ of the other nodal points is taken at the position which $\widetilde{\mathrm{B}}_{i}^{j}$ occupies after adding an appropriate small shift to $\widetilde{\mathrm{B}}_{i}{ }^{j}$. (This shift is chosen so that $(4 \cdot 1)$ may hold.)

With each $d_{j}$, the mapping $F_{j}$ from $T_{0}$ into $\mathbb{R}^{2}$ are associated as follows:

$$
F_{j}=\sum_{i=1}^{k} \mathrm{~B}_{i}^{j} \widehat{\psi}_{i} .
$$

Let $\left\{K_{j}\right\}_{j=1}^{N_{T}}$ be the set of finite elements, where

$$
K_{j}=F_{j}\left(T_{0}\right),
$$

and $\Omega_{n}$ be an approximate domain of $\Omega$ such that

$$
\Omega_{h}=\text { an interior of } \bigcup_{j=1}^{N T} K_{j} .
$$

Remark 4.1. For interior elements, it is clear that

$$
F_{j}=\mathrm{B}_{1}{ }^{j} \eta+\mathrm{B}_{2}{ }^{j}(1-\xi-\eta)+\mathrm{B}_{3}{ }^{j} \xi \text { and } K_{j}=\Delta_{j} .
$$

Therefore, the Jacobians of $F_{j}$ for interior elements are constant.

Let $S\left(\Omega_{h}\right)$ be a $N_{1}$ dimensional subspace of $H^{1}\left(\Omega_{h}\right)$ spanned by 
$\left\{\hat{\phi}_{i}(x, y)\right\}_{i=1}^{N_{1}}$. Here

$$
\widehat{\phi}_{i}=\sum_{l=1}^{h} \hat{\emptyset}\left(\mathrm{P}_{i}, \mathrm{~B}_{l}^{j}\right) \bar{\psi}_{l}\left(F_{j}^{-1}\right) \text { in } K_{j},
$$

where $\delta\left(\mathrm{P}_{i}, \mathrm{~B}_{l}{ }^{j}\right)=1$ if $\mathrm{P}_{i}=\mathrm{B}_{l}^{j}$ and $=0$ otherwisc. It should be noted that $\hat{\phi}_{i}$ satisfies the conditions $(2 \cdot 3),(2 \cdot 4)$ and $(2 \cdot 5)$. Let $P$ be an interpolating operator from $C\left(\bar{\Omega}_{h}\right)$ into $S\left(\Omega_{h}\right)$ defined by $(2 \cdot 8)$. As the boundary is smooth, there exists a one to one correspondence $\nu$ from $\Gamma$ onto $\Gamma_{h}$ such that $\mathrm{P}(t) \cdot \nu(\mathrm{P}(t))$ is normal to $\Gamma$ for $\mathrm{P}(t)=(X(t), Y(t))$ $\in \Gamma$. Let $(\delta(t), \varepsilon(t))$ be the components of the vector $\nu(\mathrm{P}(t))-\mathrm{P}(t)$ and $\lambda(t)$ be its length.

Then, we say that an isoparametric finite element procedure is of order $\alpha$. if the following four conditions are satisfied:

i) For any $f \in H^{\alpha+1}(\widetilde{\Omega}) \cap C(\widetilde{\Omega})$ and $i=0,1$,

$$
\|(I-P) f\|_{i, \Omega_{h}} \leqq c h^{a+1-i}\|f\|_{\alpha, 1, \Omega_{h}} .
$$

ii) For any $f \in H^{a}(\widetilde{\Omega}) \cap C(\widetilde{\Omega})$,

$$
\|(I-P) f\|_{0, a_{h}} \leqq c h^{a}\|f\|_{\alpha, a_{h}} .
$$

iii) For $i=0,1$,

$$
\left|\frac{d^{i} \lambda(t)}{d t^{i}}\right| \leqq c h^{\alpha+1-i}
$$

iv) For any $v \in H^{a+2}(\check{\Omega})$,

$$
\|(I-P) v\|_{0, r_{h}} \leqq c h^{n+1} \sum_{|\beta| \leqq \alpha+1}\left\|D^{\beta} v\right\|_{0, \Gamma_{h}} .
$$

Next, we define the lumping operator $Q$. Let $\left\{\bar{\psi}_{i}(\xi, \eta)\right\}_{i=1}^{k}$ be a set of real measurable functions defined in $T_{0}$ such that

i) $\bar{\phi}_{i} \in L^{2}\left(T_{0}{ }^{\circ}\right)$,

ii) $\quad \sum_{i=1}^{k} \bar{\psi}_{i} \equiv 1$ almost cverywhere in $T_{0}$

and

iii) the set $\left\{\mathrm{A}_{i} ; \mathrm{A}_{i}\right.$ is on the side of $T_{0}$ and $\left.\bar{\psi}_{i} \neq 0\right\}$ is symmetric with respect to the barycenter.

Renumbering the nodal points which belong to

$$
\left\{\mathrm{P}_{i} ;{ }^{\exists} \mathrm{B}_{l}{ }^{j} \text { such that } \mathrm{B}_{l}{ }^{j}=-\mathrm{P}_{i} \text { and } \bar{\psi}_{l} \not \equiv 0, i=1, \cdots, N_{1}\right\} \text {, }
$$

we obtain $\left\{Q_{i}\right\}_{i=1}^{N_{2}}$. Let $T\left(\Omega_{h}\right)$ be a subspace of $L^{2}\left(\Omega_{h}\right)$ spanned by $\left\{\bar{\phi}_{i}(x, y)\right\}_{i=1}^{N_{2}}$, where 


$$
\bar{\phi}_{i}=\sum_{l=1}^{k} \delta\left(Q_{i}, B_{l}^{j}\right) \bar{\phi}_{l}\left(F_{j}^{-1}\right) \text { in } K_{j} .
$$

$T\left(\Omega_{h}\right)$ satisfies the condition $(2 \cdot 6)$ and $(2 \cdot 7)$. Let $Q$ be a linear operator from $C\left(\bar{\Omega}_{h}\right)$ into $T\left(\Omega_{h}\right)$ defined by $(2 \cdot 9)$. We call $Q$ a lumping operator of type $(\beta, \gamma)$ associated with $P$, if the following two conditions are satisfied:

i) For any $f \in H^{i}(\widetilde{\Omega}) \cap C(\widetilde{\Omega})$,

$$
\|(I-Q) f\|_{0, \Omega_{h}} \leqq c h^{i}\|f\|_{i, \Omega_{h}}
$$

are satisfied for $i=1$ if $\beta=0$ and $i=1,2$ if $\beta \geqq 1$.

ii) There exists a continuous linear operator $R_{o}$ from $C\left(T_{0}\right)$ into $C\left(T_{0}\right)$ such that,

a) for $u, v \in C\left(T_{0}\right)$,

$$
\left(R_{0} v,\left(P_{0}-Q_{0}\right) u\right)_{T_{0}}=0 \text {, }
$$

where $P_{0} u=\sum_{i=1}^{k} u\left(\mathrm{~A}_{i}\right) \widehat{\psi}_{i}$ and $Q_{0} u=\sum_{i=1}^{k} u\left(\mathrm{~A}_{i}\right) \bar{\psi}_{i}$,

b) for any $f \in H^{\beta+1}(\widetilde{\Omega}) \cap C(\widetilde{\Omega})$,

$$
\|(I-R) f\|_{0, s_{h}} \leqq c h^{\beta+1}\|f\|_{\beta+1, s_{h}},
$$

where $R$ is a linear mapping from $C\left(\bar{\Omega}_{h}\right)$ into $L^{2}\left(\Omega_{h}\right)$ such that

and

c) there exists a continuous function $H_{j}(\xi, \eta)$ for each boundary element $K_{j}$ such that

$$
\left|H_{j}(\xi, \eta)\right| \leqq c h h^{r+8}
$$

and

$\left(\left(J_{j}(\xi, \eta)-H_{j}(\xi, \eta)\right) R_{0} v,\left(P_{0}-Q_{0}\right) u\right)_{T_{0}}=0$ for $u, v \in C\left(T_{0}\right)$, where $J_{j}(\xi, \eta)$ is the Jacobian of $F_{j}$.

Theorem 4.1. In (3.6) and (3.7), we use the isoparametric finite element procedure of order $\alpha(\geqq 1)$ and the lumping operator of type $(\beta, \gamma)(\beta \geqq 0)$. Assuming $f \in C(\bar{\Omega}), g \in C(\Gamma)$ and $(3 \cdot 1)$, we take $f_{A}=P f$ and $g_{A}=P g$. Then (3.6) and (3.7) has a unique solution and the following estimates hold:

$$
\|\hat{u}-\widetilde{u}\|_{1, \Omega_{h}} \leqq c\left\{h^{\alpha}\left(\|u\|_{\alpha+2, \Omega}+\|u\|_{4, \Omega}\right)+h^{\beta+2}\|u\|_{\beta+3, \Omega}+h^{\gamma+5 / 2}\|u\|_{4, \Omega}\right\}
$$


and

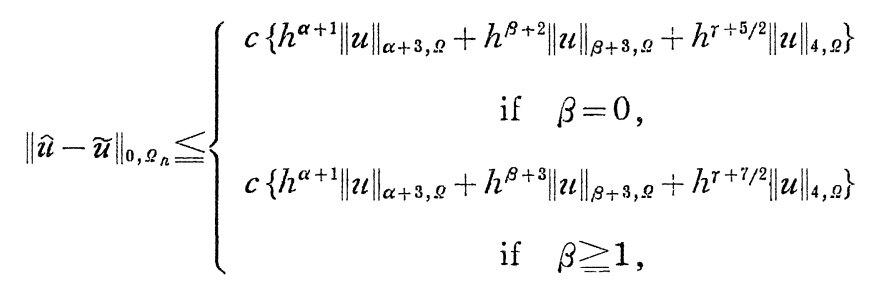

where $\widetilde{u}$ is a smooth extension over $\widetilde{\Omega}$ of the smooth solution $u$ of $(2 \cdot 1)$.

Remark 4.2. From the condition v) of $\widehat{\psi}_{i}, P g$ is well-defined on $\Gamma_{h}$.

Remark 4.3. If $Q=P, Q$ is of type $(\alpha, \infty)$. This is shown by taking $R_{0}=P_{0}$ and $H_{j} \equiv 0$. Then, $(4 \cdot 12)$ and $(4 \cdot 13)$ are reduced to

$$
\|\widehat{u}-\widetilde{u}\|_{1, \Omega_{n}} \leqq c h^{\alpha}\left(\|u\|_{\alpha+2, \Omega}+\|u\|_{4, \Omega}\right)
$$

and

$$
\|\widehat{u}-\widetilde{u}\|_{0, \Omega_{l}} \leqq c h^{\alpha+1}\|u\|_{\alpha+3, \Omega} .
$$

Proof of Theorem 4.1. We can estimate each term of the righthand side of $(3 \cdot 8)$ as follows:

$$
\left|k_{1}\right| \leqq c h^{\alpha \cdot 1}\|z\|_{1}
$$

$$
\|P \widetilde{u}-\widetilde{u}\|_{1, a_{n}} \leqq c h^{\alpha}\|u\|_{a+1},
$$

$$
\left|k_{2}\right| \leqq c\left\{h^{\alpha}\|u\|_{\alpha+2}+h^{\alpha+1}\|u\|_{4}\right\},
$$

$$
\left\|f_{A}+\Delta \widetilde{u}\right\|_{0, \Omega_{h}} \leqq c h^{\alpha}\|z\|_{\alpha+2} \text {, }
$$

$$
\|Q(\widehat{\imath}-P \widetilde{\imath})\|_{0, \Omega_{h}} /\|\widehat{\imath}-P \widetilde{u}\|_{1, \Omega_{n}} \leqq c,
$$$$
\left|(\Delta \widetilde{u},(I-Q)(\hat{u}-P \widetilde{u}))_{h}\right| /\|\widehat{u}-P \widetilde{u}\|_{1, o_{h}} \leqq c\left\{h^{\beta+2}\|u\|_{\beta-3}\right.
$$

$$
\left.+h^{\tau+\tilde{j} / 2}\|u\|_{1}\right\}
$$

and

$$
\left\|g_{A}-\frac{d \widetilde{u}}{d n_{l}}\right\|_{0, \Gamma_{h}} \leqq c h^{\alpha}\|u\|_{\alpha+2}
$$

(4.12) follow's from these inequalities. $(4 \cdot 15)$ and $(4 \cdot 17)$ are direct consequences of $(4 \cdot 1)$ and $(4 \cdot 2) .(4 \cdot 14)$ and $(4 \cdot 18)$ are proved easily. 
For $(4 \cdot 16)$, it holds that

$$
\begin{gathered}
k_{2}=\left\{\left(f_{A}+\Delta \widetilde{u}, 1\right)_{h}-\int_{\Omega-\Omega_{h}} f d x d y-\int_{\Omega_{h}-\Omega} \Delta \widetilde{u} d x d y\right. \\
\left.+\left[g_{A}, 1\right]_{h}-[g, 1]\right\} / \operatorname{mes}\left(\Omega_{h}\right) .
\end{gathered}
$$

The first three terms of the right-hand side of (4.21) are bounded by $c h^{\alpha}\|u\|_{\alpha+2}, c h^{\alpha+1}\|u\|_{3}$ and $c h^{\alpha+1}\|u\|_{3}$. To estimate the last two terms we use the functions $\delta(t)$ and $\varepsilon(t)$. From $(2 \cdot 2)$, it holds that

$$
\delta(t)=\lambda(t) \dot{Y}(t) \quad \text { and } \quad \varepsilon(t)=-\lambda(t) \dot{X}(t) .
$$

We introduce $v(x, y)$ which is defined in $\widetilde{\Omega}$ as follows: In a neighbour. hood of $\Gamma$,

$$
v(x, y)=\frac{\partial \widetilde{u}}{\partial x}(x, y) \dot{Y}(t)-\frac{\partial \widetilde{u}}{\partial y}(x, y) \dot{X}(t),
$$

where $(x-X(t), y-Y(t))$ is the normal to $\Gamma$, and $v\left(x, y^{\prime}\right)$ is extended smoothly over $\widetilde{\Omega}$. It is easy to see that $v(x, y)$ is equal to $g$ on $\Gamma$. Now we have

$$
\begin{aligned}
& {[P g, 1]_{h}-[g, 1]=[(P-I) v, 1]_{h}}
\end{aligned}
$$

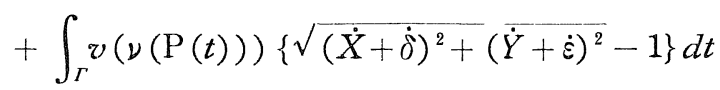

$$
\begin{aligned}
& +\int_{\Gamma}\{v(\nu(\mathrm{P}(t)))-v(\mathrm{P}(t))\} d l .
\end{aligned}
$$

Using the following inequalities,

$$
\begin{aligned}
& \left|[(P-I) v, 1]_{h}\right| \leqq c\|(P-I) v\|_{1, \Omega_{h}} \leqq c h^{\alpha}\|u\|_{\alpha-2} \\
& \left|\sqrt{(\dot{X}+\dot{\delta})^{2}+(\dot{Y}+\bar{\varepsilon})^{2}}-1\right| \leqq c h^{\alpha+1}
\end{aligned}
$$

and

$$
\begin{aligned}
|v(\nu(\mathrm{P}(t)))-v(\mathrm{P}(t))| & \leqq\left|\left\{\frac{\partial \widetilde{u}}{\partial x}(X+\delta, Y+\varepsilon)-\frac{\partial \widetilde{u}}{\partial x}(X, Y)\right\} \dot{Y}\right| \\
& +\left|\left\{\frac{\partial \widetilde{u}}{\partial y}(X+\delta, Y+\varepsilon)-\frac{\partial \widetilde{u}}{\partial y}(X, Y)\right\} \dot{X}\right|,
\end{aligned}
$$

we estimate each term of the right-hand side of (4.23) by $c h^{\alpha}\|\imath\|_{\alpha+2}$, $c h^{\alpha+1}\|u\|_{s}$ and $c h^{\alpha+1}\|u\|_{4}$. For (4.19), 


$$
\begin{gathered}
(J \widetilde{u},(I-Q)(\widehat{u}-P \widetilde{u}))_{h}=((I-R) \Delta \widetilde{u},(I-Q)(\widehat{u}-P \widetilde{u}))_{h} \\
+(R \Delta \widetilde{u},(I-Q)(\hat{u}-P \widetilde{u}))_{h} .
\end{gathered}
$$

Each term of $(4 \cdot 24)$ is estimated as follows:

$$
\begin{aligned}
\mid 1 \text { st term } \mid & \leqq\|(I-R) \Delta \widetilde{u}\|_{0, \Omega_{n}}\|(I-Q)(\grave{\imath}-P \widetilde{u})\|_{0, a_{h}} \\
& \leqq c h^{\beta-2}\|\imath\|_{\beta+3}\|\widehat{u}-P \widetilde{u}\|_{1, \Omega_{h}}
\end{aligned}
$$

and

$$
\begin{aligned}
& |2 \mathrm{nd} \operatorname{term}|=\left|\sum \int_{K_{j}} R \Delta \widetilde{u} \cdot(P-Q)(\hat{u}-P \widetilde{u}) d x d y\right| \\
& =\left|\sum \int_{T_{0}} R_{0} \Delta \widetilde{u} \cdot\left(P_{0}-Q_{0}\right)(\widehat{u}-P \widetilde{u}) J_{j}(\xi, \eta) d \xi d \eta\right| \\
& =\left|\sum_{B} \int_{T_{0}} R_{0} \Delta \widetilde{\pi} \cdot\left(P_{0}-Q_{0}\right)(\widehat{\imath}-P \widetilde{u}) H_{j}(\xi, \eta) d \xi d \eta\right| \\
& \leqq \frac{1}{\sqrt{ } \overline{2}} \max \left(\left|R_{0} \Delta \widetilde{u}\right| \frac{H_{j}(\xi, \eta)}{\sqrt{ } J_{j}(\xi, \eta)} \mid\right) \\
& \left.\times \Sigma_{B}\left\{\int_{T_{0}}\left|\left(P_{0}-Q_{0}\right)(\widehat{u}-P \widetilde{u})\right|^{2} J_{j}(\xi, \eta) d \xi d \eta\right)\right\}^{1 / 2} \\
& \leqq c\|u\|_{H} h^{r^{+}}\left\{\sum_{B} 1\right\}^{1 / 2}\|(P-Q)(\widehat{\imath}-P \widetilde{u})\|_{0, g_{h}} \\
& \leqq c\|u\|_{\mathfrak{l}} h^{\gamma-5 / 2}\|\widehat{u}-P \widetilde{u}\|_{1, \Omega_{h}},
\end{aligned}
$$

where $\sum_{B}$ means the summation over all the boundary elements. Here we have used

$$
\left|J_{j}(\xi, \eta)\right| \geqq c h^{2} \text { and }\left\{\sum_{B} 1\right\} \leqq c h^{-1} .
$$

(4.19) follows from $(4 \cdot 24),(4 \cdot 25)$ and $(4 \cdot 26)$. For $(4 \cdot 20)$, with $(4 \cdot 1)$ and $v(x, y)$ of $(4 \cdot 22)$, we obtain

$$
\begin{aligned}
\left\|g_{A}-\frac{d \pi}{d n_{h}}\right\|_{0, \Gamma_{h}} & \leqq\|P v-v\|_{0, \Gamma_{h}}+\mid v-\frac{d \pi}{d n_{h}} \|_{n, \Gamma_{h}} \\
& \leqq c\left\{h^{\alpha}\|u\|_{\alpha+2}+h^{\alpha}\|u\|_{3}\right\} .
\end{aligned}
$$

Let us prove $(4 \cdot 13)$. This is concluded from (3.22) with (4.12), $(4 \cdot 20)$ and the following estimates:

$$
\begin{aligned}
& \|P \widetilde{\widetilde{v}}-\widetilde{\boldsymbol{w}}\|_{1, \Omega_{h}} /\|\widetilde{\boldsymbol{w}}\|_{2, \Omega_{h}} \leqq c h, \\
& \left\|f_{A}+\sqrt{u}\right\|_{0, \Omega_{h}} \leqq c h^{\alpha+1}\|u\|_{\alpha-3} \text {, }
\end{aligned}
$$




$$
\left|k_{2}\right| \leqq c h^{\alpha+1}\left\{\|u\|_{\alpha^{+3}}+\|u\|_{4}\right\},
$$

$$
\|Q \widetilde{w}\|_{0, \Omega_{h}} /\|\widetilde{\boldsymbol{w}}\|_{2, \Omega_{h}} \leqq c,
$$

$$
\left|(\Delta \widetilde{u},(P-Q) \widetilde{\mathcal{v}})_{h}\right| /\|\widetilde{\mathcal{v}}\|_{2, a_{h}}
$$

$$
\leqq \begin{cases}c\left\{h^{\beta+2}\|u\|_{\beta+3}+h^{r+5 / 2}\|u\|_{4}\right\} & \text { if } \quad \beta=0, \\ c\left\{h^{\beta+3}\|u\|_{\beta+3}+h^{\gamma+7 / 2}\|u\|_{4}\right\} & \text { if } \quad \beta \geqq 1,\end{cases}
$$$$
\left|\left[g_{A}-\frac{d \widetilde{u}}{d n_{h}}, \widetilde{w}\right]_{h}\right| /\|\widetilde{w}\|_{2, \Omega_{h}} \leqq c h^{\alpha+1}\|u\|_{\alpha+3},
$$

$$
J_{1} /\|\widetilde{\mathscr{v}}\|_{2, \Omega_{h}} \leqq c h^{(\alpha+1) / 2}\|e\|_{1, g_{h}},
$$$$
J_{2} \leqq c h^{(\alpha+1) / 2}\|e\|_{1, \Omega_{h}},
$$

and

$$
\left|k_{3}\right| \leqq c h^{a+1}\left\{\|e\|_{1, \Omega_{h}}+\|u\|_{1}\right\} .
$$

$(4 \cdot 28),(4 \cdot 29),(4 \cdot 31)$ and $(4 \cdot 34) \sim(4 \cdot 36)$ are shown easily.

is proved by the same way as (4.19) in the case $\beta=0$. In $\beta \geqq 1$, using the inequality

$$
\begin{aligned}
\|(P-Q) \widetilde{w}\|_{0, g_{h}} & \leqq\|(I-P) \widetilde{\mathfrak{w}}\|_{0, \Omega_{h}}+\|(I-Q) \widetilde{w}\|_{0, s_{h}} \\
& \leqq c h^{2}\|\widetilde{\mathfrak{U}}\|_{2, g_{h}},
\end{aligned}
$$

we obtain $(4 \cdot 32)$. For $(4 \cdot 33)$, it holds that

$$
\begin{aligned}
& {\left[g_{A}-\frac{d \widetilde{u}}{d n_{h}}, \widetilde{w}\right]_{h}=\left\{\left[g_{A}, \widetilde{\mathcal{v}}\right]_{h}-[g, r v]\right\}} \\
& \quad+\left\{(-\Delta \widetilde{u}, \widetilde{w})_{h}-(f, \tau)\right\}+\left\{-a_{h}(\widetilde{u}, \widetilde{w})+a(u, w)\right\} .
\end{aligned}
$$

The second and the third terms are bounded by $c h^{a+1}\|\imath\|_{s}\|\widetilde{\mathfrak{v}}\|_{1, a_{n}}$ and $c h^{\alpha+1}\|\boldsymbol{u}\|_{2}\|\tilde{\mathfrak{w}}\|_{2, a_{n}}$, respectively. The first term is divided into

$$
[(P-I) v, \widetilde{\mathfrak{v}}]_{h}+\left\{[v, \widetilde{\mathfrak{v}}]_{h}-[v, \tau v]\right\} .
$$

The second term of $(4 \cdot 38)$ is bounded by $c h^{a+1}\|\imath\|_{4}\|\widetilde{\mathfrak{v}}\|_{2, \Omega_{h}}$ similarly in $(4 \cdot 23)$. For the first term of $(4 \cdot 38)$, using (4.4), we obtain

$$
\begin{aligned}
\left|[(P-I) v, \widetilde{\mathfrak{v}}]_{h}\right| & \leqq\|(P-I) v\|_{0, \Gamma_{h}}\|\widetilde{\mathfrak{w}}\|_{0, \Gamma_{h}} \\
& \leqq c h^{\alpha+1} \sum_{|\beta| \leqq \alpha \mid 1}\left\|D^{\beta} v\right\|_{0, \Gamma_{h}}\|\widetilde{\mathfrak{v}}\|_{0, \Gamma_{h}} \\
& \leqq c h^{\alpha+1}\|\imath\|_{\alpha+3, s}\|\widetilde{\mathfrak{w}}\|_{1, s_{h}} .
\end{aligned}
$$


From these estimates follows (4.33). Using the same argument as $(4 \cdot 39)$ to estimate the first term of the right-hand side of $(4 \cdot 23)$, we obtain $(4 \cdot 30)$. This completes the proof.

\section{§.. Examples}

Now let us consider some examples of $P$ and $Q$.

Example 5. 1. Let $k=3$, and $\left\{\widehat{\psi}_{i}\right\}_{i=1}^{3}$ be taken as follows:

$$
\begin{aligned}
& \hat{\psi}_{1}=\eta, \\
& \hat{\psi}_{2}=1-\bar{s}-\eta,
\end{aligned}
$$

and

$$
\widehat{\psi}_{3}=\xi
$$

These are the usual piecervise linear bases. $(4 \cdot 1) \sim(4 \cdot 4)$ are satisfied for $\alpha=1$. Then, three kinds of $Q$ may be considered. The first $\left\{\bar{\psi}_{i}\right\}_{i=1}^{3}$ is as follows:

$$
\begin{aligned}
& \bar{\psi}_{1}=1 \text { in } S_{1} \text { and }=0 \text { othervise, } \\
& \bar{\psi}_{2}=1 \text { in } S_{2} \text { and }=0 \text { othervise, }
\end{aligned}
$$

and

$$
\bar{\psi}_{\mathrm{j}}=1 \text { in } S_{3} \text { and }=0 \text { otherwise, }
$$

where $S_{1}$ is the quadrilateral with rertices $A_{1}, A_{4}, G$ and $A_{6}$, and $S_{2}$ and $S_{3}$ are taken similarly. (See Fig. 5. 1.) The second case is:

$$
\bar{\psi}_{1}=\bar{\psi}_{2}=\bar{\psi}_{3}=\frac{1}{3}
$$

In these cases, $Q$ is of type $(0, \infty)$ by taking

$$
R_{0} u=2 \int_{T_{0}} u(\xi, \eta) d \tilde{\xi} d \eta
$$

In fact, as $J_{i}(\xi, \eta)$ is constanl for each $j$ and

$$
\left(1, \hat{\psi}_{i}-\bar{\psi}_{i}\right)_{T_{0}}=0 \text { for } i=1,2,3,
$$

we niay take $I_{\iota_{j}}^{I}(\xi, \eta) \equiv 0$. Therefore, we obtain

$$
\|\widehat{u}-\widetilde{u}\|_{1, \Omega_{h}}=O(h) \quad \text { and } \quad\|\widehat{\imath}-\widetilde{u}\|_{0, a_{h}}=O\left(h^{2}\right) .
$$

The third case is: 


$$
\bar{\psi}_{i}=\widehat{\psi}_{i} \quad \text { for } \quad i=1,2,3 .
$$

Then $(5 \cdot 3)$ is valid from $\left(4 \cdot 12^{\prime}\right)$ and $\left(4 \cdot 13^{\prime}\right)$.

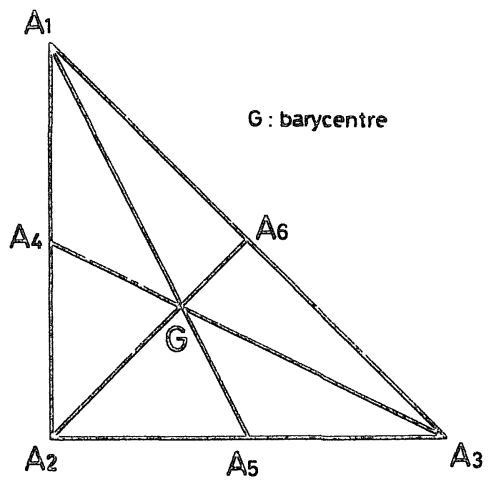

Fig. 5. 1. Fundamental Triangle.

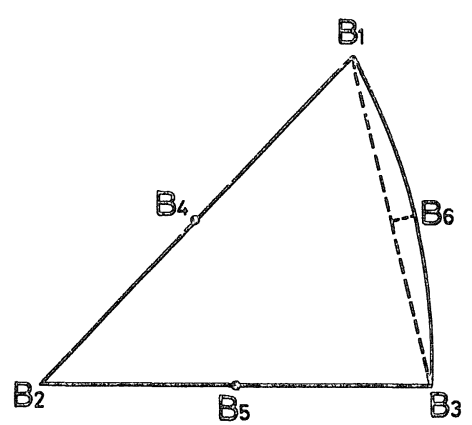

Fig. 5. 2. Curved Element.

Exæmple 5. 2. Let $k=6$. Let $\mathrm{A}_{4}, \mathrm{~A}_{5}$ and $\mathrm{A}_{6}$ be taken at the midpoints of $\mathrm{A}_{1} \mathrm{~A}_{2}, \mathrm{~A}_{2} \mathrm{~A}_{3}$ and $\mathrm{A}_{3} \mathrm{~A}_{1}$, respectively. $\left\{\widehat{\psi}_{i}\right\}_{i=1}^{6}$ is as follows:

$$
\begin{aligned}
& \widehat{\psi}_{1}=\eta(2 \eta-1), \\
& \widehat{\psi}_{2}=(1-\xi-\eta)\{1-2(\xi+\eta)\}, \\
& \widehat{\psi}_{3}=\xi(2 \xi-1), \\
& \widehat{\psi}_{4}=4 \eta(1-\xi-\eta), \\
& \widehat{\psi}_{5}=4 \xi(1-\xi-\eta),
\end{aligned}
$$

and

$$
\widehat{\psi}_{6}=4 \xi \eta
$$

These are the usual piecewise quadratic bases. $B_{6}{ }^{j}$ is taken on $\Gamma$ in such a way that $\overline{B_{6}^{j} \widetilde{B}_{6}^{j}}$ is perpendicular to $\overline{B_{3}^{j} B_{1}^{j}}$. Then, $(4 \cdot 1) \sim(4 \cdot 4)$ are satisfied for $\alpha=2$. (See [6] for (4.1) and (4.2).) The Jacobian of $F_{j}$ for the boundary element $K_{j}$ is

$$
J_{j}(\xi, \eta)=c_{0}+c_{1} \xi+c_{2} \eta,
$$

where

$$
\begin{aligned}
& c_{0}=\left(x_{3}-x_{2}\right)\left(y_{1}-y_{2}\right)-\left(x_{1}-x_{2}\right)\left(y_{3}-y_{2}\right), \\
& c_{1}=4\left\{\left(x_{3}-x_{2}\right)\left(y_{6}-\frac{y_{1}+y_{3}}{2}\right)-\left(x_{6}-\frac{x_{1}+x_{3}}{2}\right)\left(y_{3}-y_{2}\right)\right\},
\end{aligned}
$$




$$
c_{2}=4\left\{\left(x_{6}-\frac{x_{1}+x_{3}}{2}\right)\left(y_{1}-y_{2}\right)-\left(x_{1}-x_{2}\right)\left(y_{6}-\frac{y_{1}+y_{3}}{2}\right)\right\} .
$$

and $\left(x_{i}, y_{i}\right)$ is the coordinates of $B_{i}{ }^{j}$ for $i=1, \cdots, 6$. Four kinds of $Q$ are considered. In the first case $\left\{\bar{\psi}_{i}\right\}_{i=-1}^{6}$ is as follows:

$$
\begin{aligned}
& \bar{\psi}_{1}-\bar{\psi}_{2}=\bar{\psi}_{3}-0, \\
& \bar{\psi}_{4}=1 \text { in } S_{1} \text { and }=0 \text { otherwise, } \\
& \bar{\psi}_{5}=1 \text { in } S_{5} \text { and }=0 \text { otherwise, }
\end{aligned}
$$

and

$$
\bar{\phi}_{6}=1 \text { in } S_{6} \text { and }=0 \text { otherwise, }
$$

where $S_{4}$ is the triangle with vertices $A_{1}, A_{2}$ and $G$, and $S_{5}$ and $S_{6}$ are raken similarly. In the second calse,

$$
\bar{\psi}_{1}=\bar{\psi}_{2}=\bar{\psi}_{3}=0 \quad \text { and } \quad \bar{\psi}_{4}=\bar{\psi}_{5}=\bar{\psi}_{6}=\frac{1}{3}
$$

In these cases, talsing $R_{0}$ of $(5 \cdot 1)$ and $H_{j}=c_{1} 5+c_{2} \eta$ from $(5 \cdot 4)$ for the boundary element, we find $Q$ to be of tyje $(0,0)$. Therefore, we obtain

$$
\|\widehat{u}-\widetilde{u}\|_{1, \Omega_{h}}=O\left(h^{2}\right) \text { and }\|\widehat{u}-\widehat{\imath}\|_{0, \Omega_{h}}=O\left(h^{2}\right) .
$$

In the third case,

$$
\begin{aligned}
& \bar{\psi}_{1}=-\frac{1}{5}+\frac{3}{5} \eta, \\
& \bar{\psi}_{2}=\frac{2}{5}-\frac{3}{5} \xi-\frac{3}{5} \eta, \\
& \bar{\psi}_{3}=-\frac{1}{5}+\frac{3}{5} \xi, \\
& \bar{\psi}_{4}=\frac{3}{5}-\frac{4}{5} \xi, \\
& \bar{\psi}_{5}=\frac{3}{5}-\frac{4}{5} \eta,
\end{aligned}
$$

and

$$
\bar{\psi}_{6}=-\frac{1}{5}+\frac{4}{5} \xi+\frac{4}{5} \eta
$$

Taking $R_{0}$ equal to $P_{0}$ of Example 5.1 , and $H_{j}$ being the same as the 
first and the second cases, we find $Q$ to be of type $(1,0)$. This is shown by $(5 \cdot 2)$ and

$$
\left(\xi, \widehat{\psi}_{i}-\bar{\psi}_{i}\right)_{T_{0}}=0 \text { and } \quad\left(\eta, \hat{\psi}_{i}-\bar{\psi}_{i}\right)_{T_{0}}=0 \quad \text { for } \quad i=1, \cdots, 6 .
$$

Therefore, we obtain

$$
\|\widehat{u}-\widetilde{u}\|_{1, \Omega_{h}}=O\left(h^{2}\right) \quad \text { and } \quad\|\widehat{u}-\widetilde{u}\|_{0, \Omega_{h}}=O\left(h^{3}\right) .
$$

In the fourth case,

$$
\bar{\psi}_{i}=\widehat{\psi}_{i} \quad \text { for } \quad i=1, \cdots, 6 .
$$

From $\left(4 \cdot 12^{\prime}\right)$ and $\left(4 \cdot 13^{\prime}\right)$, we obtain $(5 \cdot 7)$.

\section{Acknowledgement}

The author wishes to thank Professor M. Yamaguti of Kyoto University for his continuous encouragement. This work was supported by the Sakkokai Foundation.

\section{References}

[1] Friedrichs, K. O. and Keller, H. B., A finite difference scheme for generalized Neumann problem, Numerical solution of partial differential equations, ed. by J. H. Bramble, Academic Press, 1966, 1-19.

[2] Nitsche, J., Über ein Variationprinzip zur Lösung von Dirichlet Problemen bei Verwendung von Teilräumen, die keinen Randbedingungen unterworfen sind, Abh. Math. Sem. Univ. Hamburg, 36 (1970), 9-15.

[3] - On Dirichlet problems using subspaces with nearly zero boundary conditions, The mathematical foundations of the finite element method with applications to partial differential equations, ed. by A. K. Aziz, Academic Press, 1972, 603-627.

[4] Ciarlet, P. G. and Raviart, P. A., The combined effect of curved boundaries and numerical integration in isoparametric finite elements, Ibid., 409-474.

[5] - General Lagrange and Hermite interpolation in $R^{n}$ with applications to finite element methods, Arch. Rational Mech. Anal., 46 (1971), 177-199.

[6] - - - Interpolation theory over curved elements, with applications to finite element methods, Computer Methods in Appl. Mech. and Eng., 1 (1972), 217-249.

L7] Strang, G. and Berger, A. E., The change in solution due to change in domain, Partial differential equations, AMS, 1973, 199-205.

[8] Strang, G. and Fix, G., An analysis of the finite element method, Prentice-Hall, 1973.

[9] Fujii, H., Finite-element Galerkin method for mixed initial-boundary value problems in elasticity theory, CNA-34, Center for numerical analysis, Univ. Texas (1971).

[10] Aubin, J. P., Approximation of elliptic boundary-value problerns, Wiley, 1972.

[11] Bramble, J. H. and Hilbert, S. R., Bounds for a class of linear functionals with applications to Hermite interpolation, Numer. Math., 16 (1971), 362-369.

[12] Tabata, M., Numerical experiments of higher-order approximate solutions of Neumann problems, Seisan-kenkyu, 27 (1975), 65-71, Inst. of Industrial Science, Univ. Tokyo. 